\title{
KINETIC THEORY OF ALFVÉN WAVE HEATING
}

\author{
S. M. Mahajan \\ Fusion Research Center, Dept. of Physics \\ University of Texas \\ Austin, TX 78712
}

\section{Abstract}

In a magnetohydrodynamic description of a plasma, the shear Alfvén wave is characterized by a continuous spectrum (the MHD continuum) which results from a singularity at $\omega \simeq k_{\|}(r) V_{A}(r)$, where $\omega$ is the frequency, $k_{\|}$is the wave, number along the direction of the magnetic field and $\mathrm{V}_{\mathrm{A}}=\mathrm{B}_{0} /(4 \rho \pi)^{1 / 2}$ is the Alfvén speed, and $\mathrm{r}$ is the direction of inhomogeniety. The associated electromagnetic fields become large at this resonance layer [in fact, in ideal MHD they become singular], and if some dissipation is allowed, the transfer of energy from the waves to the electrons can take place. These continuum modes are localized in space, and can be effective in heating plasmas in narrow regions around the resonanct surface. Recently, ${ }^{1-3}$ a new class of nonsingular global eigenmodes of the plasma have been discovered which are wide spread in the plasma, and could effectively transfer energy to the bulk of the plasma. These modes arise because of a strong coupling between the shear and the evanascent compressional mode. The coupling is provided by gradients of density, equilibrium current and nonideal effects like finite $\omega / \omega_{c i}$, where $\omega_{c i}$ is the ion cyclotron frequency. In order to understand the effectiveness of these modes to heat plasmas, i.e., to determine the effective impedance, the energy deposition profiles, parametric dependence of the heating efficiency, etc., a kinetic theory with the electron parallel dynamics is needed. We have developed a detailed kinetic theory to study the structure of the continuum as well as global eigenmodes in an inhomogenous current carrying plasma. The global modes are comparatively much less damped than the continuum modes (which become discrete in the kinetic theory) and hence are easier to excite. The implication, of course, is that the large part of energy transfer in a Alfvén Wave experiment would be mediated through these global modes. This theoretical conjecture is already confirmed in laboratory experiments on PRETEXT Tokamak.

I will present the basic theoretical analysis as well as discuss the implications of these findings.

M. R. Kundu and G. D. Holman (eds.). Unstable Current Systems and Plasma Instabilities in Astrophysics, $391-392$. (c) 1985 by the IAU. 


\section{References}

1. D.W. Ross, G.L., Chen, and S.M. Mahajan, "Kinetic description of Alfven wave heating", Phys. Fluids 25, 652 (1982).

2. S.M. Mahajan, D.W. Ross and G-L. Chen, "Discrete Alfven Eigenmode Spectrum in Magnetohydrodynamics", The University of Texas, Fusion Research Center Report 非 249.

3. S.M. Mahajan, "Kinetic Theory of Alfven Waves", The University of Texas, Institute for Fusion Studies Report 非84. 